\title{
José Luis Domínguez Álvarez (2021), Comunidades discriminadas y territorios rurales abandonados. Políticas públicas y Derecho administrativo frente a la despoblación
}

\author{
Nora Libertad Rodríguez Peña \\ Universidad de Salamanca (España) \\ ORCID: https://orcid.org/0000-0001-6282-7570 \\ nrodriguezp@usal.es
}

\begin{abstract}
NOTA BIOGRÁFICA
Licenciada en Derecho por la Universidad Centroamérica, Máster en Democracia y buen Gobierno por la Universidad de Salamanca y Doctoranda en el Programa de Doctorado en Administración, Hacienda y Justicia en el Estado Social de la Universidad de Salamanca. Personal Investigador en Formación (FPU) adscrita al Área de Derecho Financiero y Tributario del Departamento de Derecho Administrativo, Financiero y Procesal de la Facultad de Derecho de la Universidad de Salamanca. Miembro del Grupo de Investigación Reconocido TRIBUTUM. Dentro de sus líneas de investigación destaca la necesaria inclusión del Derecho Financiero y Tributario dentro de las políticas públicas encaminadas a combatir la despoblación del medio rural español.
\end{abstract}

\section{RESUMEN}

Recensión de la monografía de José Luis Domínguez Álvarez: Comunidades discriminadas y territorios rurales abandonados. Políticas públicas y Derecho administrativo frente a la despoblación, Cizur Menor, Thomson Reuters-Aranzadi, 2021. 297 pp.

\section{PALABRAS CLAVE}

Desarrollo rural sostenible; políticas públicas; reto demográfico; Derecho Administrativo; España vaciada.

\begin{abstract}
Book Review of author José Luis Domínguez ÁlvAREZ: Comunidades discriminadas y territorios rurales abandonados. Políticas públicas y Derecho administrativo frente a la despoblación, Cizur Menor, Thomson Reuters-Aranzadi, 2021, 297 pp.
\end{abstract}

\section{KEYWORDS}

Sustainable rural development; public policies; demographic challenge; Administrative Law; emptied Spain.

En los últimos años, la agenda pública española ha puesto de relieve que afrontar las distintas dimensiones del reto demográfico exige concebirlo como una auténtica Política de Estado, que demanda un entendimiento transversal de la acción de gobierno y el compromiso y la acción coordinada de todas las Administraciones públicas. El reto demográfico y la lucha contra la despoblación del medio rural español se configuran así, como una cuestión de lucha contra la desigualdad y la injusticia ${ }^{1}$, sobre la que ya desde 2006 hacía hincapié la Unión Europea (UE), convirtiéndose, en consecuencia, en uno de los ejes prioritarios que

1 Al respecto son ilustrativas las palabras de Fernando Pablo (2020, pp. 19-27). 
estructuran el Plan de Recuperación, Transformación y Resiliencia, el "Plan España Puede", destinado a ejecutar políticas públicas bajo el amparo económico de los Fondos Next Generation UE.

Como bien apunta el profesor Marcos M. Fernando Pablo, Catedrático de Derecho Administrativo de la Universidad de Salamanca quien con sobrada maestría elaboró la presentación de la monografía que reseñamos, existe una estrecha relación entre el Derecho, como instrumento de ordenación de la sociedad, y la aceleración del fenómeno de despoblación de las áreas rurales. De ahí que afirme que la despoblación rural es «[u]n problema de personas; un problema de territorios; un problema, en primer lugar, jurídico, pues a ese campo pertenece la regulación de las relaciones no solo entre personas, sino también entre poderes públicos y ciudadanos, y entre personas y medio» (Fernando Pablo, 2019, p. 12). En torno a esta cuestión, y más concretamente, en torno a la necesidad de revigorizar el papel protagonista del Derecho administrativo como herramienta de vanguardia en la lucha contra el vaciamiento poblacional de las áreas rurales, versa la última monografía ahora recensionada de José Luis Domínguez Álvarez, obra en la que se pone de manifiesto el amplio compromiso del autor y del Área de Derecho Administrativo de la Universidad de Salamanca con la promoción de políticas públicas incardinadas a garantizar el cumplimiento de una verdadera Estrategia Nacional frente al reto demográfico que garantice no solo el desarrollo rural sostenible, sino también, el impulso de la cohesión social y territorial del Estado².

En ese sentido, la publicación que estas líneas reseñan titulada Comunidades discriminadas y territorios rurales abandonados. Politicas públicas y Derecho administrativo frente a la despoblación denota entre sus páginas el arduo compromiso investigador y el ímpetu innovador del autor al constituirla, en esencia, en un código de propuestas jurídico-administrativas que ponen el foco de atención en cómo afrontar desde la Administración pública la dificultosa tarea de revitalizar la España rural, obra que incluso se adelanta en el tiempo a la aprobación por parte del Gobierno de la nación de las 130 medidas frente al reto demográfico del Plan de Recuperación que incluye en sus ejes de actuación el impulso de la transición ecológica; la plena conectividad territorial; el desarrollo e innovación en el territorio; el impulso del turismo sostenible; la igualdad de derechos y oportunidades de las mujeres y los jóvenes del medio rural; el fomento del emprendimiento y de la actividad empresarial; la garantía y el refuerzo de los servicios públicos esenciales; el bienestar social, económico y cultural de los habitantes del medio rural; y, como no podía ser de otra manera, el impulso de reformas normativas e institucionales para abordar el Reto demográfico desde las distintas Administraciones públicas.

Bajo ese espíritu, esta obra evita conscientemente la realización de un extenso análisis acerca de la reconstrucción de las causas que propiciaron la quiebra generalizada de las sociedades rurales tradicionales ${ }^{3}$. No se trata, en definitiva, de una descripción más sobre la magnitud del desastre, o un alegato nostálgico sobre el empobrecimiento natural y cultural que conlleva el declive rural. La finalidad que se pretende es bien distinta, hacer un llamamiento a las Administraciones públicas, como garantes, aunque no en solitario, de los intereses generales de la ciudadanía para la articulación y transformación urgente de las políticas públicas de desarrollo rural sostenible; la importancia de garantizar la actividad prestacional de las Administraciones públicas en las áreas rurales; la reconstrucción de la idea de servicio público mediante la vuelta a la idea larvada en los viejos postulados de la Escuela de Burdeos como presupuesto para poner fin a la desconexión digital de los territorios rurales; el empoderamiento de la mujer rural como agente indispensable para para alcanzar el ansiado desarrollo rural sostenible; la incorporación de los Objetivos de Desarrollo Sostenible (ODS) y la Agenda 2030 para el Desarrollo Sostenible como palanca transformadora para devolver la dignidad a los pueblos; la revitalización de las Entidades locales como elemento institucional esencial para combatir la despoblación; la urgencia de avanzar en el establecimiento de un ordenamiento jurídico adaptado a las necesidades y particularidades de las comunidades rurales y el impulso de la esperada simplificación administrativa como mecanismo para dinamizar las economías locales; el alcance real de las medidas tributarias frente al vaciamiento de los territorios rurales; o la importancia capital de fomentar la cooperación transfronteriza. Estas son solamente algunas de las paradas obligatorias en las que se detiene esta obra con el firme propósito de proponer políticas públicas destinadas a garantizar la pervivencia de las comunidades rurales y devolver el alma y la dignidad a la población que loablemente habita en las áreas rurales despobladas.

Este planteamiento lleva al autor, irremediablemente, a constatar una de las primeras evidencias empíricas, que justifica en gran medida el devenir del estudio: «el vaciamiento y la consiguiente sangría de-

2 Sobre este tema en particular véanse las líneas escritas por el autor de la monografía objeto de esta recensión en Domínguez Álvarez (2020a, pp. 217-243).

3 Este tema fue brillantemente tratado con anterioridad por el autor de la monografía recensionada en Domínguez Álvarez (2019a, pp. 21-36). 
GAPP. Nueva Época - N. ${ }^{0}$ 27, noviembre 2021 - ISSN: 1989-8991 - DOI: https://doi.org/10.24965/gapp.i27.10949 - [Págs. 146-151]

José Luis Domínguez Álvarez: Comunidades discriminadas y territorios rurales abandonados. Políticas públicas y Derecho..

Nora Libertad Rodríguez Peña

mográfica de las áreas rurales obedece a algo más que la yuxtaposición de intereses y prioridades propias de la (i)lógica economía de mercado. La existencia de una normativa administrativa desmedida, el acusado intervencionismo al que las Administraciones públicas someten cualquier iniciativa vital en el medio rural, o la desaparición progresiva de los servicios públicos básicos -imprescindibles para garantizar unos estándares mínimos de bienestar y calidad de vida-, son algunas de las razones que se esconden tras el drama de la despoblación y que amenazan, en gran medida, la pervivencia de las comunidades rurales».

Bajo esta premisa, la obra invita al lector a emprender una travesía a través del estudio de algunos de los hitos jurídico-administrativos más relevantes en la materia para otorgar respuestas tangibles y realistas a buena parte de los interrogantes que envuelven a la problemática del reto demográfico y la despoblación de las áreas rurales, ofreciendo al lector un manifiesto de políticas públicas por un medio rural en positivo y con futuro, espíritu del que hacen eco las palabras del Prólogo "El medio rural en el centro de las políticas públicas. Del reto demográfico a la cohesión territorial" elaborado por Francesc Boya Alós, actual Secretario General para el Reto Demográfico -institución que, integrada en la Vicepresidencia Cuarta del Gobierno de España, se encarga del impulso de la acción administrativa en materia de despoblación-, en el que se pone de relieve la conexión entre los postulados que desarrolla el autor en esta monografía con las líneas de actuación estratégica de la Administración General del Estado y la UE.

Por consiguiente, en el primer capítulo, el autor parte de un análisis detallado de los hitos normativos que han vertebrado las políticas públicas de desarrollo rural sostenible en el Estado español -el cual transcurre desde la Ley 25/1982, de 30 de junio, de agricultura de montaña, hasta los últimos avances jurídicoadministrativos en la materia-, y sienta las bases para afianzar la necesidad y la urgencia de adoptar una auténtica Estrategia Nacional frente al reto demográfico que permita afrontar la problemática de la despoblación que el autor concibe como «un auténtico problema de Estado a caballo entre la igualdad territorial, la dignidad de la población y el interés general de las Administraciones públicas».

De ahí, que el segundo capítulo se nos presente con un análisis pormenorizado de la evolución de las políticas públicas en materia de desarrollo rural sostenible, en el que se pone de relieve la importancia capital que posee la actuación y el liderazgo de la UE, al tiempo que se constatan una serie de debilidades y carencias esenciales que impregnan el impulso de la política española de desarrollo rural (dificultades en los procesos de ejecución y justificación de fondos europeos, ausencia de coordinación entre las diferentes esferas administrativas, excesiva burocratización de los grupos de acción local, entre otras). De igual forma, permite al lector realizar una primera incursión en el estudio de la importancia que el despliegue del Plan de Recuperación, Transformación y Resiliencia "Plan España Puede" y el impulso del Instrumento de Recuperación Europea poseen en la tediosa tarea de materializar la inversión económica estatal y comunitaria a las áreas rurales despobladas.

En el capítulo tercero, el autor focaliza sus esfuerzos en clarificar, lejos de planteamientos preconcebidos y argumentos tautológicos, la importancia de garantizar la actividad prestacional de las Administraciones públicas en las áreas rurales, elemento que de la mano de la elaboración de políticas públicas innovadoras se presenta como «presupuesto indispensable para combatir la despoblación». De esta forma, se analiza la precaria situación que atraviesa la prestación de determinados servicios esenciales en el medio rural espa$\tilde{n ̃ o l}^{4}$, cuya escasez destaca "como una de las principales debilidades de las zonas rurales a revitalizar». En este sentido, cabe mencionar el derecho fundamental a la educación ${ }^{5}$, la sanidad rural, o el sistema de transportes y la dotación de infraestructuras, los dos primeros claves para el mantenimiento y la fijación de población, y los últimos en la garantía de la custodia del territorio ${ }^{6}$. De igual forma, en este capítulo se contempla una serie de importantes reflexiones en torno a la regeneración urbana y el auge de la función social de la propiedad, las cuales se presentan como herramientas esenciales para mitigar la crisis de la vivienda en la España rural, cuestión que además se incluye como una de las medidas del eje 1 del Plan de Recuperación.

Por su parte, el capítulo cuarto, profundiza en la desconexión tecnológica del medio rural, otra de las realidades que impide el brote del emprendimiento digital y el empleo del teletrabajo en estos territorios, y que el Plan de Recuperación desarrolla en el eje 2 apostando por la transición digital y la plena conectividad

4 Sobre el desarrollo de políticas públicas innovadoras que garantizar el acceso a la población rural a unos servicios esenciales de calidad mediante el uso de las nuevas tecnologías como una oportunidad sin precedentes para revitalizar las áreas rurales despobladas véase Domínguez Álvarez (2021a, pp. 91-124).

5 Este tema también ha sido desarrollado de forma previa por el autor de la monografía recensionada centrando el enfoque de su estudio en la población joven que aún reside en el medio rural en Domínguez Álvarez (2020b, pp. 60-78).

6 Sobre la custodia del territorio son ilustradoras las líneas de Fernández de Gatta Sánchez (2020, pp. 91-112). 
como un elemento vertebrador de la cohesión social y territorial. De ahí, el interés del autor por desentrañar la estrecha relación que existe entre la nueva concepción europea de los servicios de interés general y el patente retraso tecnológico que padecen estos territorios. Para ello se ahonda en el análisis de la conceptualización de las telecomunicaciones como servicios de interés económico general, las carencias que presenta la actuación prestacional de las Administraciones públicas en este sector, las deficiencias del Estado garante en la materia y la escasa efectividad de la imposición de obligaciones de servicio universal. Todo ello conduce al autor a reflexionar incluso en el papel que desempeña el emprendimiento digital y el teletrabajo como punta de lanza para promover el asentamiento de población en el mundo rural en la etapa post COVID-19 en que nos encontramos ${ }^{7}$.

El autor ofrece en el capítulo quinto un estudio de la igualdad de oportunidades en las áreas rurales, consciente de que las mujeres rurales son agentes indispensables para alcanzar el ansiado desarrollo rural sostenible ${ }^{8}$. Así, el fomento, promoción y apoyo de políticas públicas dirigidas a empoderar y visibilizar a las mujeres del medio rural constituyen el núcleo duro de este capítulo. Tras un análisis de la situación sociodemográfica de la población femenina asentada en el medio rural se esbozan una serie de propuestas innovadoras orientadas a fomentar la sensibilización y concienciación en materia de igualdad de género en las áreas rurales, impulsar la empleabilidad y la participación de las mujeres rurales en la toma de decisiones y mejorar la intervención administrativa a la hora de combatir la violencia de género en las comunidades rurales, en plena concordancia con el eje 5 del Plan de Recuperación.

Así se enlaza el capítulo seis donde el autor realiza un análisis de la incorporación de los ODS y la Agenda 2030 para el Desarrollo Sostenible en las políticas públicas orientadas a transformar la realidad rural, claves en el eje 10 del Plan de Recuperación que aborda el impulso de la Agenda 2030 a nivel local entre el conjunto de reformas normativas e institucionales destinadas a afrontar el Reto demográfico. Con esta finalidad se analiza el protagonismo que poseen la igualdad de género y la despoblación como objetivos prioritarios de desarrollo sostenible ${ }^{9}$.

Por su parte, el capítulo séptimo, aborda uno de los elementos nucleares de la obra, el cual ha sido -y sigue siendo- objeto de excelsos debates dentro de la doctrina iuspublicista, la revitalización de las Entidades locales, la cual se presenta en esta ocasión como elemento institucional esencial para combatir la despoblación ${ }^{10}$. Para ello, la obra se introduce en el azaroso mundo del tejer y destejer normativo que ha caracterizado la evolución del régimen local en las últimas décadas, destacando el papel básico que la Administración local juega en la prestación de servicios esenciales y escudriñando los perniciosos efectos que la promulgación de la Ley $27 / 2013$, de 27 de diciembre, de Racionalización y Sostenibilidad Local y la adopción de otra serie de medidas de exacerbado control de la actuación local han propiciado en la erosión de la garantía institucional de la autonomía local, por un lado, y por otro, en la acentuación de los procesos de abandono de los territorios rurales, lo cual se refleja en la disminución drástica del padrón de habitantes, otro de los aspectos sometido a análisis. De igual forma, se analiza la necesidad de impulsar «la renovada actividad de fomento, "nudge"», como alternativa cierta de la Administración local para la consecución de objetivos sociales en las áreas despobladas ante la constante constricción de su esquema competencial.

En el capítulo octavo el autor completa el corazón de esta obra al dedicarse a examinar la acuciante necesidad de promover el establecimiento de un ordenamiento jurídico adaptado a las necesidades y a las concretas particularidades de las comunidades rurales que instaure la creación de políticas públicas dirigidas a poner en valor el resurgir del medio rural, su conectividad y su sostenibilidad. Así, en primer lugar, se examinan las elevadas cotas de ineficacia que provoca la «paradoja de la sobrerregulación de la realidad rural», y los beneficios que comporta «legislar mejor», eliminar obstáculos burocráticos y apostar por la celeridad administrativa, como premisas esenciales para impulsar la actividad económica y el asentamiento de población en las áreas rurales. En segundo lugar, individualiza el estudio de la simplificación administrativa y la consolidación de la administración electrónica como condiciones indispensables para la promoción del

7 En este particular también véase la reciente publicación del autor de la monografía recensionada Domínguez Álvarez (2021c, pp. 389-398).

8 La mujer como vector de cambio del medio rural ha sido defendido en múltiples ocasiones por el autor de la monografía objeto de recensión en Domínguez Álvarez (2019b, pp. 377-397); Domínguez Álvarez (2019c, pp. 173-189). También véanse los trabajos de González Bustos (2020a, pp. 167-182) y González Bustos (2020b).

9 En este sentido también véase Domínguez Álvarez (2020c, pp. 127-146).

10 Algunas de las cuestiones sobre las que el autor de la monografía recensionada ya había trabajado con anterioridad, pueden verse en Domínguez Álvarez (2021b, 73-120) y Domínguez Álvarez y Buenaventura Calvo (2020, pp. 145-165). También véase Terrón Santos y Alonso Martín (2020, pp. 183-200). 
GAPP. Nueva Época - N. ${ }^{2}$ 27, noviembre 2021 - ISSN: 1989-8991 - DOI: https://doi.org/10.24965/gapp.i27.10949 - [Págs. 146-151] José Luis Domínguez Álvarez: Comunidades discriminadas y territorios rurales abandonados. Políticas públicas y Derecho...

emprendimiento de políticas públicas y la dinamización de la economía rural. Y, en tercer lugar, destaca la necesaria incorporación de medidas de lucha contra la despoblación rural en las políticas financieras y tributarias que deben estar incardinadas a paliar el déficit de inversión pública del que adolecen los territorios rurales abandonados y no en la desmembración a golpe de beneficio fiscal de nuestro sistema tributario. En este apartado el autor resalta las consecuencias funestas que comporta «el establecimiento desmedido de un sistema fiscal orientado a la "discriminación fiscal positiva" como solución a todos los males que aquejan a las áreas rurales despobladas» sin la previa consideración de los límites constitucionales y comunitarios que comporta el intento ${ }^{11}$.

Concluye esta obra el capítulo noveno que pone en valor la cooperación transfronteriza frente a la despoblación del territorio. Así, se centra en el estudio de los instrumentos de cooperación territorial europea (INTERREG) y de algunos instrumentos de reciente adopción por parte de las autoridades españolas y portuguesas, entre las que destaca la Estrategia Común de Desarrollo Transfronterizo entre España y Portugal.

En definitiva, nos encontramos ante una obra precursora en materia de despoblación del medio rural que apuesta por un estudio holístico jurídico, práctico y realista de las políticas públicas necesarias para afrontar el reto demográfico que padece nuestro país. Su originalidad se ve completada por el admirable y completo análisis bibliográfico de la despoblación de las áreas rurales que realiza el autor, quien alejado del egoísmo ególatra que muchas veces acompaña a los estudios individuales, apuesta por visibilizar la labor investigadora jurídico-administrativa de una comunidad científica que apuesta por la revitalización del medio rural desde el Derecho, desde el empleo de políticas públicas innovadoras que garanticen su futuro con impacto a corto plazo y visión a largo plazo, reconociendo, en esencia, que la hora de la España rural ha llegado porque no podemos permitirnos vivir sin territorio.

\section{REFERENCIAS BIBLIOGRÁFICAS}

Domínguez Álvarez, J. L. (2021a). Internet y nuevas tecnologías como punta de lanza para la revitalización de territorios rurales despoblados: La necesaria reconstrucción de la idea de servicio público. Revista Digital de Derecho Administrativo, (26), 91-124. Https://doi.Org/10.18601/21452946.N26.04

Domínguez Álvarez, J. L. (2021b). La importancia del municipalismo para la consecución del nuevo resurgir de la ruralidad: la disyuntiva entre devolver el alma a los pueblos o abandonar el territorio. Revista jurídica de Castilla y León, (53), 73-120. https://www.jcyl.es/web/jcyl/AdministracionPublica/es/Plantilla100Detalle/1131978346397/ Publicacion/1285024111139/Redaccion

Domínguez Álvarez, J. L. (2021c). El impulso del teletrabajo en la España vacia(da) en la era post COVID-19: Del mito al logos. En J. F. Rodríguez Ayuso y E. Atienza Macías (coords.), El nuevo marco legal del teletrabajo en España: Presente y futuro, una aproximación multidisciplinar (pp. 389-398). Wolters Kluwer.

Domínguez Álvarez, J. L. (2020a). El agotamiento de las políticas públicas relacionadas con el desarrollo rural. En M. Fernando Pablo y J. L. Domínguez Álvarez (dirs.), Rural Renaissance: derecho y medio rural (pp. 217-243). Thomson Reuters-Aranzadi.

Domínguez Álvarez, J. L. (2020b). El desigual acceso de la juventud rural a los servicios públicos: la necesidad de impulsar la educación en la España vaciada. Cuadernos de Investigación en Juventud, (8), e047. https://www.cjex. org/wp-content/uploads/2021/02/Desigual-Acceso.pdf

Domínguez Álvarez, J. L. (2020c). Objetivos de desarrollo sostenible, igualdad de género y despoblación: una respuesta desde las administraciones públicas. En A. Figueruelo Burrieza y M. del Pozo Pérez (dirs.), (Des) igualdad y violencia de género: el nudo gordiano de la sociedad globalizada (pp. 127-146). Thomson ReutersAranzadi.

Domínguez Álvarez, J. L. y Buenaventura Calvo, A. (2020). ¿Reinventarse o morir?. El municipalismo como piedra angular del anhelado desarrollo rural sostenible. San Martín del Castañar, una referencia al sur de la provincia de Salamanca. En M. Fernando Pablo y Domínguez Álvarez, J. L. (dirs.), Rural Renaissance: derecho y medio rural (pp. 145-165). Thomson Reuters-Aranzadi.

Domínguez Álvarez, J. L. (2019a). La despoblación en Castilla y León: políticas públicas innovadoras que garanticen el futuro de la juventud en el medio rural. Cuadernos de Investigación en Juventud, (6), e028. https://www.cjex.org/ wp-content/uploads/2021/02/Despoblacio\%CC\%81n-CyL.pdf

Domínguez Álvarez, J. L. (2019b). Despoblación e igualdad: el difícil papel de la mujer en el medio rural. En L. Bujosa Vadell y M. del Pozo Pérez (dirs.), Proceso penal y víctimas especialmente vulnerables: aspectos interdisciplinares (pp. 377-397). Thomson Reuters-Aranzadi.

11 Sobre la inclusión cuidadosa de políticas públicas, tributarias y financieras en la lucha contra la despoblación del medio rural véase Rodríguez Peña (2020, pp. 299-320); Lago Montero (2020, pp. 65-90) e Iglesias Caridad (2020, pp. 113-143). 
Domínguez Álvarez, J. L. (2019c). Aproximación a la intervención en vulnerabilidad y exclusión social en el medio rural: una respuesta desde las entidades locales. En L. Bujosa Vadell, M. del Pozo Pérez (dirs.), Protocolos de actuación con víctimas especialmente vulnerables: una guía de buenas prácticas (pp. 173-189). Thomson Reuters-Aranzadi.

Fernández de Gatta Sánchez, D. (2020). La custodia del territorio: régimen de un instrumento complementario del desarrollo rural. En M. Fernando Pablo y J. L. Domínguez Álvarez (dirs.), Rural Renaissance: derecho y medio rural (pp. 91-112). Thomson Reuters-Aranzadi.

Fernando Pablo, M. (2019). Devolver el alma a los pueblos: el encuentro "Rural Renaissance». Ars luris Salmanticensis: revista europea e iberoamericana de pensamiento y análisis de derecho, ciencia política y criminología, 7(2), 11-13. https://revistas.usal.es/index.php/ais/article/view/22488

Fernando Pablo, M. (2020). ¡Faltan cabezas!: Una nota sobre Derecho público y ruralidad. En M. Fernando Pablo y J. L. Domínguez Álvarez (dirs.), Rural Renaissance: derecho y medio rural (pp. 19-27). Thomson Reuters-Aranzadi.

González Bustos, M. A. (2020a). El destacado papel de la mujer en el desarrollo rural. En M. Fernando Pablo y J. L. Domínguez Álvarez (dirs.), Rural Renaissance: derecho y medio rural (pp. 167-182). Thomson ReutersAranzadi.

González Bustos, M. A. (2020b). Mujer y desarrollo sostenible en el medio rural. Atelier.

Iglesias Caridad, M. (2020). El reto demográfico desde el municipalismo. Medidas tributarias locales favorecedoras del emprendimiento y del empleo. En M. Fernando Pablo y Domínguez Álvarez, J. L. (dirs.), Rural Renaissance: derecho y medio rural (pp. 113-143). Thomson Reuters-Aranzadi.

Lago Montero, J. M. (2020). El derecho financiero frente a la despoblación en el ámbito rural. En M. Fernando Pablo y Domínguez Álvarez, J. L. (dirs.), Rural Renaissance: derecho y medio rural (pp. 65-90). Thomson ReutersAranzadi.

Rodríguez Peña, N. L. (2020). ¿Es posible el establecimiento de un régimen tributario favorable para el medio rural en España? En M. Fernando Pablo y Domínguez Álvarez, J. L. (dirs.), Rural Renaissance: derecho y medio rural (pp. 299-320). Thomson Reuters-Aranzadi.

Terrón Santos, D. y Alonso Martín, J. B. (2020). La importancia de los Grupos de Acción Local para alcanzar el desarrollo rural sostenible. El caso concreto de las Sierras de Salamanca. En M. Fernando Pablo y Domínguez Álvarez, J. L. (dirs.), Rural Renaissance: derecho y medio rural (pp. 183-200). Thomson Reuters-Aranzadi. 